\title{
Vasohibin as an endothelium-derived negative feedback regulator of angiogenesis
}

\author{
Kazuhide Watanabe, ${ }^{1}$ Yasuhiro Hasegawa, ${ }^{1}$ Hiroshi Yamashita, ${ }^{1}$ Kazue Shimizu, ${ }^{1}$ Yuanying Ding, \\ Mayumi Abe, ${ }^{1}$ Hideki Ohta, ${ }^{2}$ Keiichi Imagawa, ${ }^{2}$ Kanji Hojo, ${ }^{3}$ Hideo Maki, ${ }^{3}$ \\ Hikaru Sonoda, ${ }^{2}$ and Yasufumi Sato ${ }^{1}$
}

1Department of Vascular Biology, Institute of Development, Aging, and Cancer, Tohoku University, Sendai, Japan. ²Diagnostic Science Division, ${ }^{3}$ Discovery Research Laboratories, Shionogi \& Co. Ltd., Osaka, Japan.

\begin{abstract}
Negative feedback is a crucial physiological regulatory mechanism, but no such regulator of angiogenesis has been established. Here we report a novel angiogenesis inhibitor that is induced in endothelial cells (ECs) by angiogenic factors and inhibits angiogenesis in an autocrine manner. We have performed cDNA microarray analysis to survey VEGF-inducible genes in human ECs. We characterized one such gene, KIAA1036, whose function had been uncharacterized. The recombinant protein inhibited migration, proliferation, and network formation by ECs as well as angiogenesis in vivo. This inhibitory effect was selective to ECs, as the protein did not affect the migration of smooth muscle cells or fibroblasts. Specific elimination of the expression of KIAA1036 in ECs restored their responsiveness to a higher concentration of VEGF. The expression of KIAA1036 was selective to ECs, and hypoxia or TNF- $\alpha$ abrogated its inducible expression. As this molecule is preferentially expressed in ECs, we designated it "vasohibin." Transfection of Lewis lung carcinoma cells with the vasohibin gene did not affect the proliferation of cancer cells in vitro, but did inhibit tumor growth and tumor angiogenesis in vivo. We propose vasohibin to be an endothelium-derived negative feedback regulator of angiogenesis.
\end{abstract}

\section{Introduction}

Blood vessels are one of the most quiescent tissues in the adult, but have the capacity for new vessel growth under certain conditions. A switch to the actively angiogenic phenotype is dependent upon the local balance between angiogenic factors and inhibitors (1). Angiogenic factors include VEGF and its family members FGF-2 and HGF, and inflammatory cytokines such as TNF- $\alpha$ and IL-8 (2). VEGF is the principal angiogenic factor. It is induced by hypoxia and stimulates the migration and proliferation of endothelial cells (ECs) (3). A number of angiogenesis inhibitors have also been identified. Thrombospondin-1 (TSP-1) is a large molecular multidomain glycoprotein whose expression is regulated by tumor suppressor p53 $(4,5)$. Pigment epithelium-derived factor (PEDF), a member of the serpin superfamily, which lacks inhibitory activity against either serine or cysteine proteinases, possesses antiangiogenic as well as neurotropic activities $(6,7)$. Sixteen-kDa prolactin (8), angiostatin (9), and endostatin (10) are proteolytic fragments originating from proteins without any antiangiogenic activities themselves. Several chemokines, such as growth-related oncogene- $\beta$ (11), IFN- $\gamma$ inducible protein-10 (12), and platelet factor-4 (13), also exhibit antiangiogenic activity. Although angiopoietin- 1 and angiopoietin-2 can be classified as either angiogenic factors or angiogenesis inhibitors, the main function of angiopoietins is to regulate mural cell attachment for vascular stabilization (14).

Nonstandard abbreviations used: AS-ODN, antisense ODN; CAM, chorioallantoic membrane; EBM, endothelial basal medium; EC, endothelial cell; ERK, extracellular signal-regulated kinase; Flk-1, fetal liver kinase-1; HAEC, human aortic EC; HASMC, human aortic SMC; HMVEC, human dermal microvascular EC; HUVEC, human umbilical vein EC; KDR, kinase insert domain receptor; LLC, Lewis lung carcinoma; ODN, oligodeoxynucleotide; PEDF, pigment epithelium-derived factor; PIGF, placenta growth factor; Scr-ODN, scrambled ODN; S-ODN, sense ODN; TBS, Tris-buffered saline; TSP-1, thrombospondin-1; UTR, untranslated region.

Conflict of interest: The authors have declared that no conflict of interest exists.

Citation for this article: J. Clin. Invest. 114:898-907 (2004)

doi:10.1172/JCI200421152
Negative feedback regulation is one of the most important physiological mechanisms with which bodies are endowed, and has been demonstrated to control a wide range of phenomena (15). However, no such regulators have been established for the regulation of angiogenesis to date. TSP-1 is thought to act as a barrier for angiogenesis (16). PEDF has recently been recognized as an angiogenesis inhibitor in specific organs such as the prostate and pancreas (17). Although angiostatin and endostatin inhibit tumor angiogenesis and exhibit antitumor activity (18), their physiological roles in the regulation of angiogenesis remain to be established. Inflammation does not associate with physiological angiogenesis, and thus it is unclear whether antiangiogenic chemokines play a physiological role in angiogenesis.

We hypothesized that angiogenic factors might induce bioreactive substances, including feedback regulators of angiogenesis in ECs. We therefore examined the VEGF-inducible genes in ECs. Here we characterize one such gene (KIAA1036, preferentially expressed in ECs and its effect selective to ECs, and therefore designated "vasohibin"), whose function was previously undetermined. Our results suggest that the gene product inhibits angiogenesis and functions as an endothelium-derived negative feedback regulator of angiogenesis.

\section{Results}

We searched for VEGF-inducible genes in ECs by cDNA microarray analysis (19). Among 7,267 human sequences, 97 were induced more than twofold by VEGF stimulation in human umbilical vein ECs (HUVECs) at the 24-hour time point. Of these 97 sequences, 11 were novel or uncharacterized in terms of their biological function. Here we focused on one such sequence, AF055021. An expressed sequence tag-clustered database, AssEST, indicated that AF055021 encodes the 3' untranslated region (UTR) of KIAA1036 cDNA (GenBank accession number AB028959). The KIAA1036 gene localizes in chromosome 14 and includes a 5'-UTR of 385 
bp, an open reading frame of 1,098 bp, and a 3'-UTR of 3,998 bp. Based on the sequence of the human KIAA1036 gene, we cloned a mouse counterpart of KIAA1036 cDNA. The deduced amino acid sequences of the human and mouse KIAA1036 proteins are shown in Figure 1A. A cluster of basic amino acids was present in the C-terminus region, but neither a classical secretion signal sequence nor any other functional motif was found among these amino acid sequences during the database search.

Using the coding region of the KIAA1036 gene as a probe, we performed Northern blotting. We observed a single band of KIAA1036 mRNA to be induced in HUVECs by VEGF stimulation (Figure $1 B)$. Quantitative RT-PCR revealed that the effect of VEGF on the induction of KIAA1036 was concentration-dependent (Figure 1C). The induction of KIAA1036 mRNA by VEGF stimulation was observable in ECs isolated from different sites, such as human aortic ECs (HAECs) and human dermal microvascular ECs (HMVECs) (data not shown). We prepared an $\mathrm{mAb}$ against KIAA1036 peptide. Western blotting confirmed that our anti-KIAA1036 $\mathrm{mAb}$ specifically recognized human KIAA1036 protein (Figure 1D, lane 1 and

\section{Figure 1}

KIAA1036 is an endothelium-derived VEGFinducible secretory protein. (A) The deduced amino acid sequences of the human and mouse KIAA1036 (KIAA) proteins are shown. Asterisks indicate identical amino acids between human and mouse. (B) A single KIAA1036 mRNA was induced by VEGF. HUVECs were stimulated with VEGF (1 nM) for the indicated periods and then Northern blotting was performed. (C) VEGF increased KIAA1036 mRNA in a concentration-dependent manner. HUVECs were stimulated with the indicated concentration of VEGF for 24 hours and then real-time RT-PCR was performed. (D) KIAA1036 protein was synthesized and secreted. GM7373 cells transfected with KIAA1036 gene were lysed. Equal amounts of protein were applied to lane 1 and lane 2, and transferred to the filter. The filter was then separated into 2 parts. Western blotting was performed with anti-KIAA1036 $\mathrm{mAb}$ (lane 1). Prior to Western blotting, antiKIAA1036 mAb was absorbed with antigen peptide (lane 2). HUVECs were stimulated with VEGF (1 $\mathrm{nM}$ ) for the following periods and were lysed for Western blotting: lane 3, 0 hours; lane 4, 12 hours; lane 5, 24 hours; lane 6,48 hours. HUVECs were cultured for 3 days in the growth medium and then cells were lysed for Western blotting (lane 7). After this incubation, the medium was collected and concentrated. Five hundred microliters of concentrated medium was subjected to immunoprecipitation followed by Western blotting (lane 8). Asterisk indicates protein in the medium. (E) KIAA1036 protein does not colocalize with ER. HUVECs in the growth medium were used for the immunostaining of calnexin (red) and KIAA1036 protein (green).

A

lane 2). Our anti-KIAA1036 mAb did not crossreact with bovine KIAA1036 protein upon Western blotting (data not shown). VEGF increased the synthesis of KIAA1036 protein $(42 \mathrm{kDa})$ in HUVECs in a time-dependent manner (Figure 1D, lanes 3-6). We were also able to show the presence of the KIAA1036 protein in the culture medium of HUVECs, suggesting that the protein was secreted (Figure $1 \mathrm{D}$, lane 8). The apparent lower molecular weight of vasohibin in the medium ( $30 \mathrm{kDa}$, asterisk) may indicate a cleaving process during secretion. Using calnexin as a marker of ER, we clarified the intracellular localization of KIAA1036 protein in HUVECs. It appeared that KIAA1036 protein did not correlate with ER (Figure 1E). This evidence together with the lack of classical signal sequence suggests that KIAA1036 is an unconventional secretory protein.

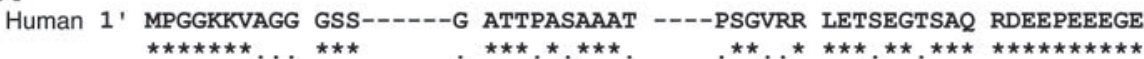
Mouse $1 "$ MPGGKKVVPS GSSSASPNAA ATTTAAAAAA AAAPHSGTKR LETTEGASAQ RDEEPEEEGE

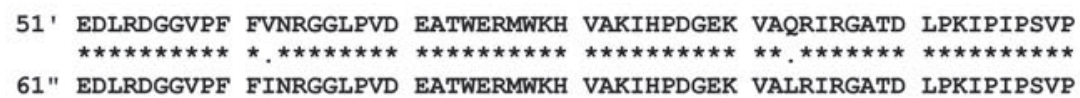

111 TFQPSTPVPE RLEAVQRYIR ELQYNHTGTQ FFEIKKSRPL TGLMDLAKEM TKEALPIKCL

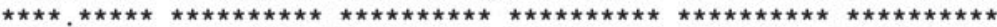
121" TFQPTTPVPE RLEAVQRYIR ELQYNHTGTQ FFEIKKSRPL TGLMDIAKEM TKEALPIKCL

171' EAVILGIYLT NSMPTLERFP ISFKTYFSGN YFRHIVLGVN FAGRYGALGM SRREDLMYKP

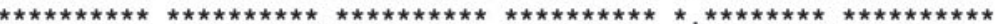

181" EAVILGIYLT NSMPTLERFP ISFKTYFSGN YFRHIVLGVN FGGRYGALGM SRREDIMYKP

231' PAFRTLSELV LDFEAAYGRC WHVLKKVKLG QSVSHDPHSV EQIEWKHSVL DVERLGRDDF

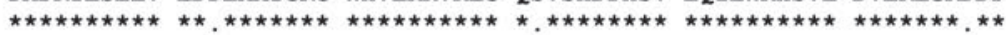
241" PAFRTLSELV IDYEAAYGRC WHVLKKVKLG QCVSHDPHSV EQIEWKHSVL DVERLGREDF

291 RKELERHARD MRLKIGKGTG PPSPTKDRKK DVSSPQRAQS SPHRRNSRSE RRPSGDKKTS

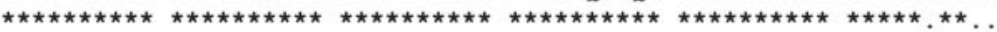

301" RKELERHARD MRLKIGKGTG PPSPTKDRKK DVSSPQRAQS SPHRRNSRSE RRPSGEKKPA

351' EPKAMPDLNG YQIRV

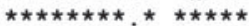

$361 "$ EPKAMPDLSG YQIRV

B

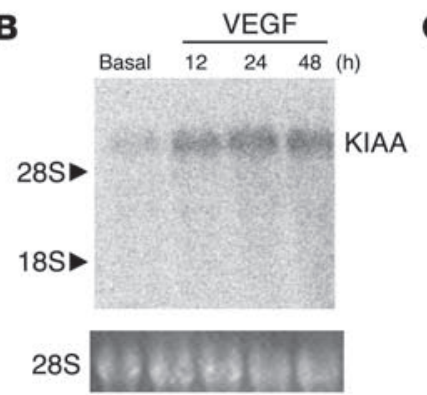

D

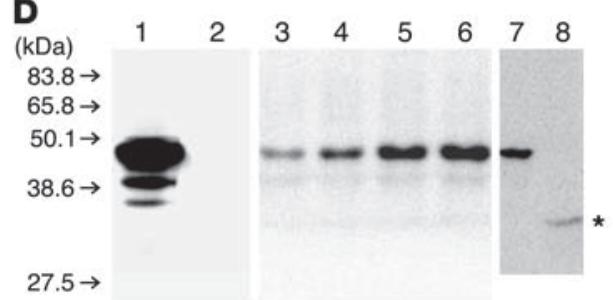

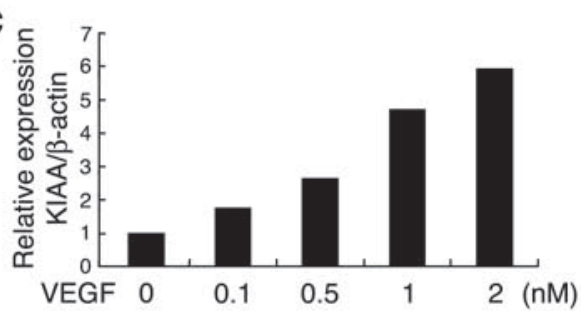

E

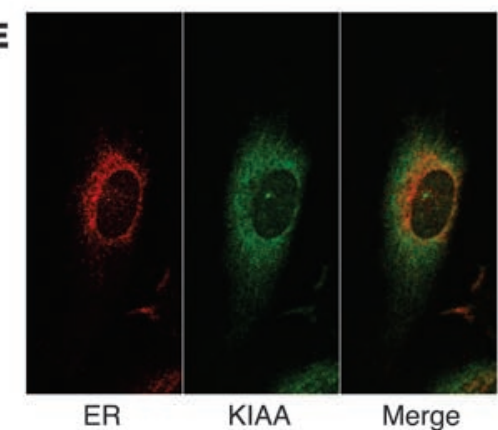




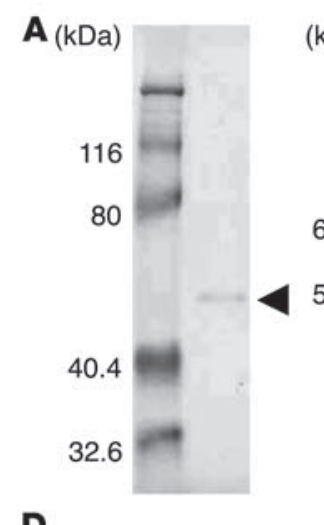

D
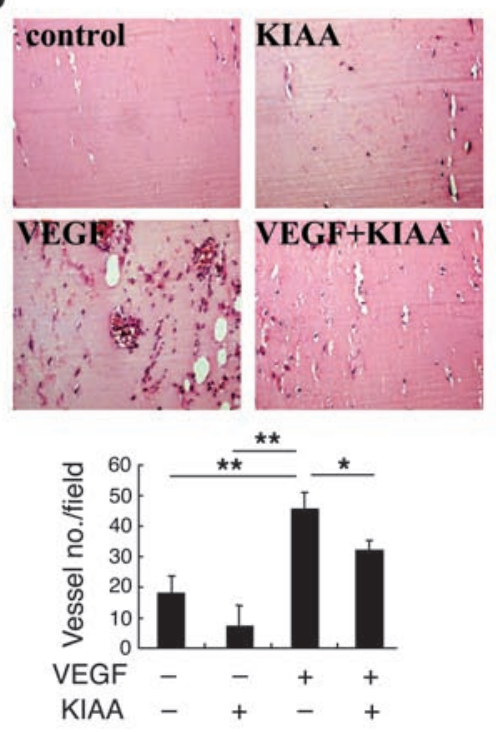

B
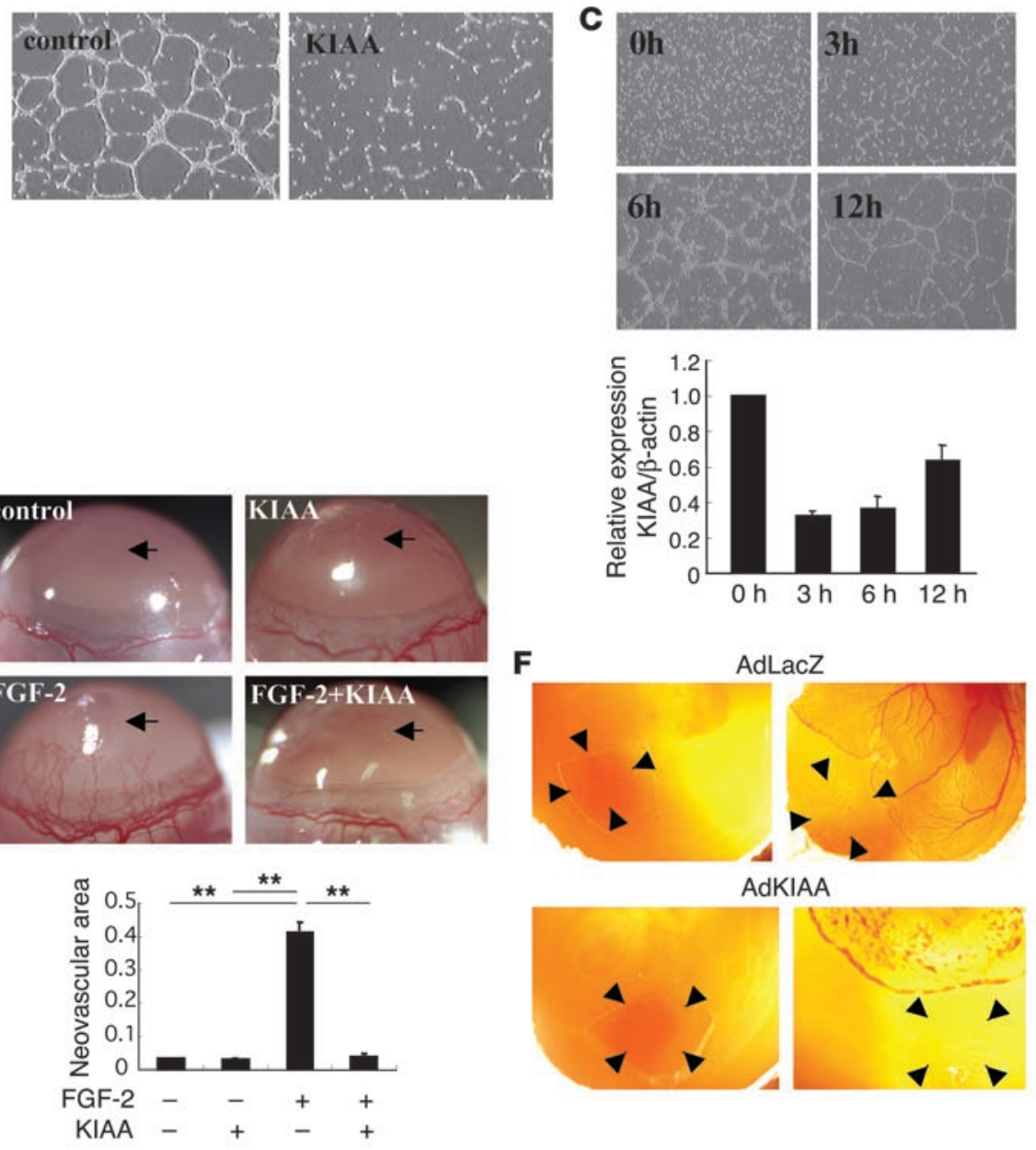

$\mathbf{F}$
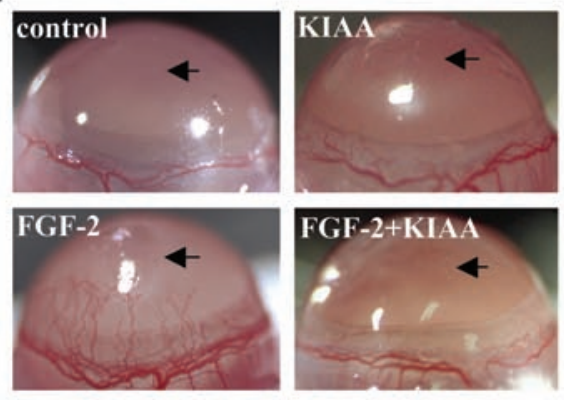

$\mathbf{E}$

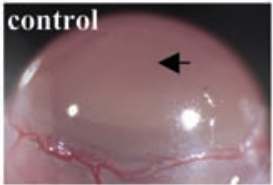

AdKIAA

\section{Figure 2}

KIAA1036 inhibits angiogenesis. (A) Preparation of KIAA1036 protein. SDS-PAGE/Coomassie brilliant blue staining is shown on the left and Western blotting on the right. Arrows indicate KIAA1036. (B) Effect of KIAA1036 on network formation by ECs. HUVECs were plated on Matrigel in the absence or presence of KIAA1036 (10 nM). (C) Downregulation of KIAA1036 during network formation on Matrigel. HUVECs were plated on Matrigel, and after the indicated period of incubation, total RNA was obtained and real-time RT-PCR was performed. Values are expressed as mean \pm SD of 3 samples. (D) Matrigel implantation analysis was performed as described in Methods. The vessel number per low-power field in 3 different fields was counted for each sample. Values are expressed as mean \pm SD of 5 animals. (E) Mouse corneal micropocket assay was performed as described in Methods. Arrows indicate the site where pellets were implanted. Neovascular area $\left(\mathrm{mm}^{2}\right)$ was determined using NIH Image. Values are expressed as mean \pm SD of 5 eyes. (F) CAM assay using adenovirus vectors was performed as described in Methods. The nylon mesh containing adenovirus/Matrigel mixture was placed on the peripheral zone of the CAM, where vascular structure would not appear (left panels). Four days after adenovirus infection, vascular formation was evaluated by macroscopic observation (right). Arrowheads indicate the site where the nylon mesh was placed. ${ }^{*} P<0.05,{ }^{* \star} P<0.01$.

We next prepared recombinant KIAA1036 protein to examine whether the KIAA1036 protein exhibited any biological effects. Our preparation of KIAA1036 protein is shown in Figure 2A. HUVECs spontaneously form network-like structures when plated on Matrigel. When the recombinant KIAA1036 protein was added to the medium, the protein inhibited this network formation (Figure $2 \mathrm{~B}$ ). This result suggests that KIAA1036 protein has antiangiogenic activity. Interestingly, the expression of endogenous KIAA1036 in HUVECs was found to be downregulated when Matrigel-plated HUVECs formed these network-like structures (Figure 2C). It was then further examined by 3 independent assays whether KIAA1036 exhibited antiangiogenic activities in vivo. Matrigel was mixed with VEGF and/or KIAA1036 protein and injected subcutaneously into mice. Seven days later, the Matrigel was removed, fixed, and sectioned for histological analysis. It was revealed that KIAA1036 had inhibited VEGFstimulated new vessel formation in Matrigel (Figure 2D). A mouse corneal micropocket assay was performed as described in Methods. KIAA1036 protein apparently inhibited FGF-2-stimulated angiogenesis in mouse cornea (Figure 2E). We introduced the KIAA1036 gene into a replication-defective adenovirus vector and used it in a chicken chorioallantoic membrane (CAM) assay. The adenovirus vector encoding KIAA1036 (AdKIAA) abrogated vessel formation whereas the control adenovirus vector encoding $\beta$-gal (AdLacZ) did not (Figure 2F). These 3 independent assays confirm the antiangiogenic activity of KIAA1036 protein. We used the same protein in Matrigel implantation and the corneal micropocket assay. However, the inhibition seen in the Matrigel 
A

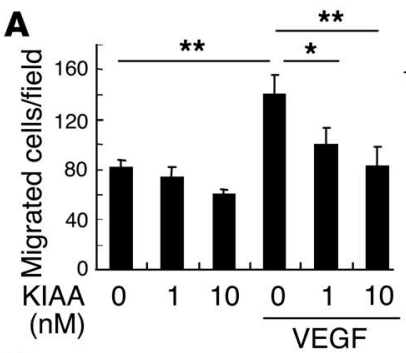

C

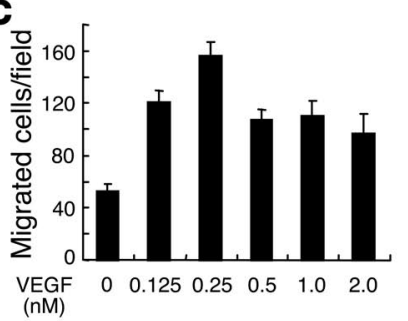

E
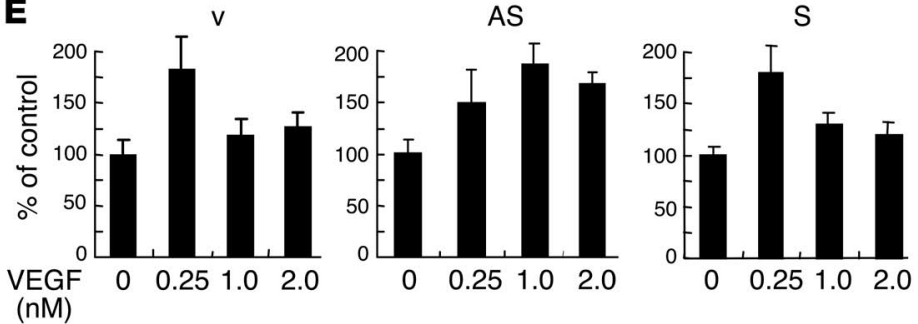

D

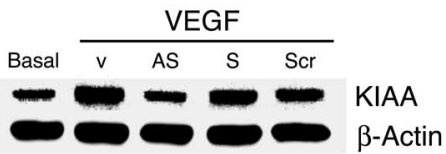

Figure 3

KIAA1036 may act as a negative feedback regulator. (A) Effect of KIAA1036 on the migration of HUVECs. Migration of HUVECs was analyzed as described in Methods. The indicated concentrations of growth factors and/or KIAA1036 protein were placed in the lower chamber of the Transwell insert. Values are expressed as mean \pm SD of 4 samples. (B) Effect of KIAA1036 on the migration of HASMCs or fibroblasts. Migration of HASMCs or fibroblasts was analyzed as described in Methods. The indicated combinations of PDGF, FGF-2, and KIAA1036 protein were placed in the lower chamber. Values are expressed as mean \pm SD of 4 samples. (C) Bell-shaped pattern of the VEGFstimulated migration of HUVECs. The indicated concentrations of VEGF were placed in the lower chamber and HUVECs were plated in the upper chamber. Values are expressed as mean \pm SD of 4 samples. (D) Selective downregulation of KIAA1036 synthesis. HUVECs were incubated for 4 hours with $500 \mathrm{nM}$ synthetic phosphorothioate ODNs or vehicle alone. Thereafter, HUVECs were stimulated with VEGF (1 nM) for 24 hours, and Western blotting for KIAA1036 was performed. AS, AS-ODN; S, S-ODN; Scr, Scr-ODN; v, vehicle. (E) Modulation of the bell-shaped pattern of the VEGF effect by KIAA1036 AS-ODNs. HUVECs were incubated for 4 hours with $500 \mathrm{nM}$ phosphorothioate ODNs. Thereafter, HUVECs were subjected to the migration assay described above. Values are expressed as mean \pm SD of 4 samples. ${ }^{*} P<0.05,{ }^{*} P<0.01$.

implantation analysis was not remarkable compared with that in the corneal micropocket assay. Although the reason for this difference is obscure at present, one possible explanation is that Matrigel contains substances that interact with KIAA1036 protein and interfere with its effects.

The effects of KIAA1036 protein on the angiogenesis-related properties of ECs were further investigated. KIAA1036 protein inhibited VEGF- or FGF-2-stimulated migration (Figure 3A) as well as DNA synthesis in HUVECs (data not shown). In contrast, KIAA1036 protein did not inhibit PDGF-stimulated migration of human aortic SMCs (HASMCs) or FGF-2-stimulated migration of human fibroblasts (Figure 3B). Thus, the inhibitory effect of KIAA1036 protein is selective to ECs. Since KIAA1036 was induced in ECs by VEGF stimulation, we hypothesized that endogenous vasohibin might be involved in negative feedback regulation. The examination of this hypothesis proceeded as follows. The dose-response curve for the effect of VEGF on the migration of ECs exhibits a bell-shaped pattern (20). Our present study revealed that the effect of VEGF on migration peaked at $0.25 \mathrm{nM}$; higher concentrations elicited less stimulation (Figure 3C). To determine whether the loss of responsiveness to higher concentrations of VEGF is mediated by VEGFinduced expression of endogenous KIAA1036, we generated an antisense oligodeoxynucleotide (AS-ODN) that specifically inhibited the VEGFstimulated KIAA1036 protein synthesis. Neither control sense ODN (S-ODN) nor scrambled ODN (Scr-ODN) impacted this effect of VEGF (Figure 3D). We then applied ODNs to cell migration analysis. As shown in Figure 3E, AS-ODNs, but not S-ODNs or Scr-ODNs, abrogated the decrease in cell migration at $1.0 \mathrm{nM}$ VEGF and shifted the dose-response curve to the right. As VEGF induced KIAA1036 mRNA in a concentration-dependent manner (Figure 1C), this data suggests that the induced vasohibin inhibited the effect of higher-concentration VEGF as a negative feedback regulator.

The expression profile of KIAA1036 was examined in more detail. HASMCs expressed KIAA1036 weakly, and PDGF modestly increased its expression (Figure 4A). Although fibroblasts did express KIAA1036, the level of expression was very low and was unresponsive to the FGF-2 stimulation (Figure 4A). KIAA1036 expression was not observed in keratinocytes under either basal or EGF-stimulated conditions (Figure 4A). The expression of KIAA1036 in various organs in vivo was examined. Northern blotting revealed that KIAA1036 was predominantly expressed in the brain and placenta, and to a lesser extent in the heart and kidney (Figure 4B). The placenta is a site at which active vascular remodeling takes place. Immunohistochemical analysis revealed that KIAA1036 was present selectively in the endothelial layer of the human placenta (Figure 4C). KIAA1036 was also present selectively in the endothelial layer of the human brain (data not shown). Since KIAA1036 is preferentially expressed in ECs and its effect is selective to ECs, we designated KIAA1036 vasohibin. A robust expression of vasohibin was demonstrated in the developing organs of the human embryo, suggesting a role for this molecule in development (Figure 4D).

We characterized the regulation of vasohibin expression in ECs. FGF-2 induced the expression of vasohibin to a level comparable to that obtained with VEGF (Figure 5A). Placenta growth factor (PlGF) and HGF induced the expression to a lesser extent. Neither 
A

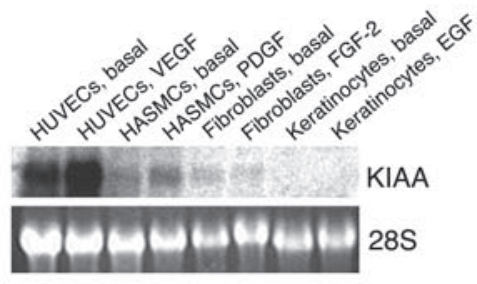

C

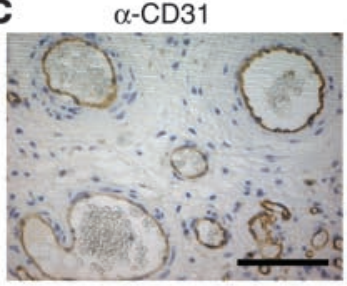

$\alpha-\mathrm{KIAA}$

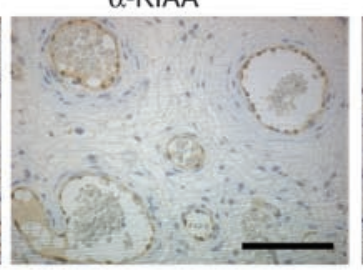

Mouse IgG

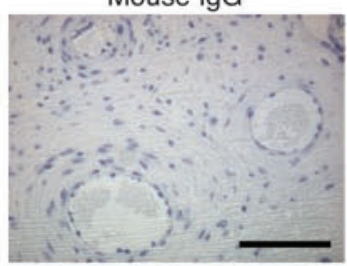

B

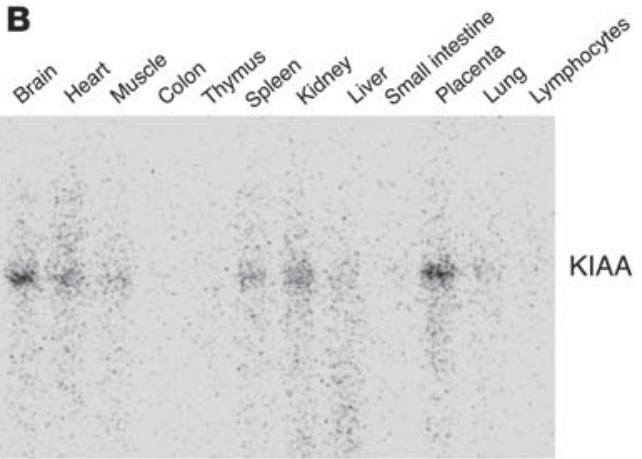

D
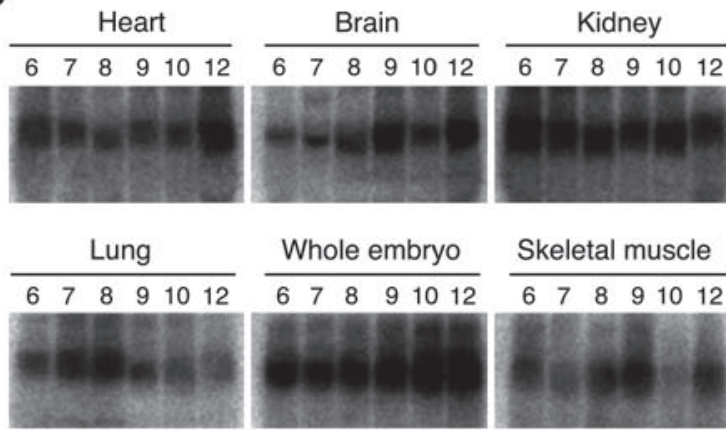

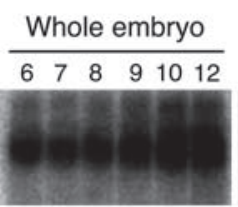

Skeletal muscle

$\begin{array}{llllllll}6 & 7 & 8 & 9 & 10 & 12 & \text { Embryonic period (wk) }\end{array}$

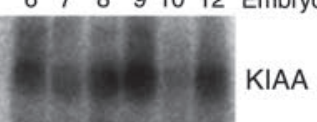

Figure 4

KIAA1036 is preferentially expressed in ECs. (A) Expression of KIAA1036 in cultured cells. Cells were preincubated in $0.1 \%$ FCS/ $\alpha$-MEM for 12 hours and then stimulated with growth factors as follows: HUVECs with VEGF (1 nM), HASMCs with PDGF (1 nM), human fibroblasts with FGF-2 (2 nM), and keratinocytes with EGF (1 nM). Thereafter, total RNA was obtained and Northern blotting for vasohibin was performed. (B) Expression of KIAA1036 in vivo was examined by multiple-tissue Northern blot. (C) Localization of KIAA1036 protein in the placenta. Sections of human placenta were subjected to immunostaining. Anti-human CD31 mAb, anti-KIAA1036 mAb, or mouse IgG was used as the primary Ab. Scale bars: $100 \mu \mathrm{m}$. (D) Expression of KIAA1036 in human embryo. Northern blotting for vasohibin was performed using a human developmental total RNA Northern blot.

angiopoietin-1 nor angiopoietin-2 affected the expression of vasohibin (data not shown). TNF- $\alpha$ alone did not significantly affect the expression of vasohibin (Figure 5, A and B), but did inhibit the VEGF-stimulated vasohibin mRNA expression and protein synthesis in ECs (Figure 5B). Hypoxia is known to act as a trigger of both physiological and pathological angiogenesis by inducing VEGF $(21,22)$. Hypoxia did not affect the expression of vasohibin (Figure 5C). However, hypoxia did inhibit VEGF-stimulated vasohibin mRNA expression, as well as vasohibin protein synthesis in ECs (Figure 5C). We next examined whether vasohibin affected VEGFmediated signaling. HUVECs infected with AdLacZ or AdKIAA were stimulated with VEGF. Infection with AdKIAA did not affect VEGF-mediated tyrosine phosphorylation of kinase insert domain receptor (KDR), a type 2 VEGF receptor, or activation of extracellular signal-regulated kinase-1/2 (ERK1/2) (Figure 5D). Treatment of HUVECs with recombinant vasohibin protein also did not affect VEGF-mediated tyrosine phosphorylation of KDR (data not shown).

Tumors are generally associated with hypoxia and the infiltration of inflammatory cells $(22,23)$. Thus, it is reasonable to hypothesize that this negative feedback system might not operate efficiently in tumors. It was therefore assumed that exogenous vasohibin would affect tumor growth and tumor angiogenesis, and we undertook experiments to determine if this were so. We transfected human vasohibin cDNA into Lewis lung carcinoma (LLC) cells, established permanent transfectants, and further isolated 2 vasohibinproducing clones, clone 16 and clone 19 (Figure 6A). Vasohibin cDNA transfection did not alter the proliferation of LLC cells in vitro (Figure 6B). To show the effect of vasohibin produced by LLC cells, we used a modified Boyden chamber. Mock- or vasohibin- transfected LLC cells were plated in the lower chamber of the Transwell insert, and the migration of HUVECs from the upper chamber toward LLC cells in the lower chamber was analyzed. As shown in Figure 6C, the number of migrated HUVECs was significantly reduced when vasohibin-transfected LLC cells were plated in the lower chamber. We then inoculated mice intradermally with LLC cells, and tumor growth was observed. As shown in Figure $6 \mathrm{D}$, tumor growth in vasohibin-producing clone 16 and clone 19 in mice was significantly retarded. Immunohistological analysis of CD31 was performed to evaluate the extent of tumor angiogenesis. It was revealed that tumors of mock transfectants contained large luminal vessels, whereas those of clone 16 and clone 19 contained very small ones, even when tumor size did not differ greatly on day 8 (Figure 6E). Quantitative analysis of vascular luminal space revealed that the vascular area within tumors was significantly decreased in clone 16 and clone 19 (Figure 6F).

\section{Discussion}

Here we isolated a novel angiogenesis inhibitor from VEGFinducible transcripts in HUVECs and designated it vasohibin. The noteworthy characteristics of vasohibin are as follows. It is selectively expressed in ECs; its expression is induced by representative angiogenic growth factors such as VEGF and FGF-2; it inhibits migration, proliferation, and tube formation by ECs in vitro and angiogenesis in vivo; the inhibitory effect of vasohibin is selective to ECs; and the specific downregulation of vasohibin expression modulates the dose-response curve of ECs to VEGF. Although a number of angiogenesis inhibitors have been reported to date, to the best of our knowledge, none has been reported to be selectively expressed in ECs and inducible by angiogenic factors. A bell-shaped 
concentration-dependent curve of the chemotactic cell migration can be explained as follows: when a higher concentration of a factor in the lower chamber of the Transwell insert is added, the diffusion across the filter may increase. Therefore, the concentration gradient between the lower and upper chambers becomes smaller. However, when the expression of vasohibin was downregulated by AS-ODN, the concentration curve shifted to the right. This result may suggest vasohibin as a candidate regulator of angiogenesis acting through endothelium-derived negative feedback.

The deduced amino acid sequences of vasohibin revealed that the N-terminal region was variable between human and mouse, but the entire amino acid sequence was highly conserved (about 94\% identical). This may indicate the physiological importance of this molecule. A cluster of basic amino acids was found in the $\mathrm{C}$-terminus region, but neither a classical secretion signal sequence nor any other functional motif was found among these amino acid sequences. The size of the protein in cell extracts evaluated by Western blotting corresponded well to the molecular weight estimated from the deduced amino acid sequence, indicating that this protein is not glycosylated. Moreover, since vasohibin does not contain a classical signal sequence, the mode of its secretion is thought to be unconventional. Indeed, vasohibin did not colocalize with ER (Figure 1E). The process of unconventional protein secretion, also known as ER/ Golgi-independent protein secretion, has recently been acknowledged. The molecular mechanisms and the molecular identities of the mechanistic components mediating this process are diverse. The apparently smaller molecular weight of vasohibin in the medium indicates that there might be a cleaving process required for the secretion. Proteolytic processing in unconventional protein secretion has been shown for 2 isoforms of IL-1, namely IL- $1 \alpha$ and IL- $1 \beta$. The processing of IL- $1 \alpha$ involves myristoylation, insertion into the plasma membrane, and calpain-dependent cleavage $(24,25)$. IL-converting enzyme processes the precursor form of IL-1 $\beta$ (26). It remains elusive whether the processing of vasohibin is analogous to either of the IL-1 isoforms.

The mechanism by which vasohibin expresses its effect on ECs is not known at the moment. Recombinant vasohibin protein inhibited VEGFstimulated and FGF-2-stimulated angiogenesis. Moreover, tumor angiogenesis was inhibited when LLC cells were transfected with vasohibin gene. These results indicate that vasohibin can act on ECs from outside. Although vasohibin inhibited FGF-2-stimulated migration of HUVECs, it did not inhibit FGF-2stimulated migration of human fibroblasts. Moreover, vasohibin did not affect the tyrosine phosphorylation of KDR or the activation of ERK1/2 when HUVECs were stimulated with VEGF. These results indicate that vasohibin does not act merely as an antagonist of growth factors. Further study is being conducted to elucidate whether or not vasohibin exhibits its effect through a specific vasohibin receptor.

Angiogenesis is involved in various physiological processes. A recent report on PEDF knockout mice indicates that $\mathrm{PEDF}$ regulates postnatal angiogenesis of specific hormone-sensitive organs such as the prostate and the pancreas (17). Angiogenesis is also required for embryonic development. However, the physiological function of angiogenesis inhibitors in embryonic development is largely unknown. Here we showed that vasohibin was expressed in ECs of placenta, where active vascular remodeling took place. Moreover, the robust expression of vasohibin was shown in developing organs in human embryo as well. These results may suggest that vasohibin acts as a physiological regulator of vascular development in the embryo.

Another important issue is the significance of angiogenesis inhibitors in pathological angiogenesis. Hypoxia and the inflammatory cytokine TNF- $\alpha$ inhibited VEGF-stimulated induction of vasohibin in ECs. Regarding hypoxia, we analyzed about $5 \mathrm{~kb}$ upstream of the $5^{\prime}$ flanking region of the vasohibin gene, but we did not find any consensus sequences of hypoxiaresponsive elements. Nevertheless, it is well known that tumors are associated with hypoxia as well as the infiltration of inflammatory cells $(22,23)$. Therefore, this negative feedback system may not operate efficiently in tumors. We hypothesized that supplemental vasohibin would be valuable in such conditions. Here we showed that transfection of vasohibin cDNA to tumor cells

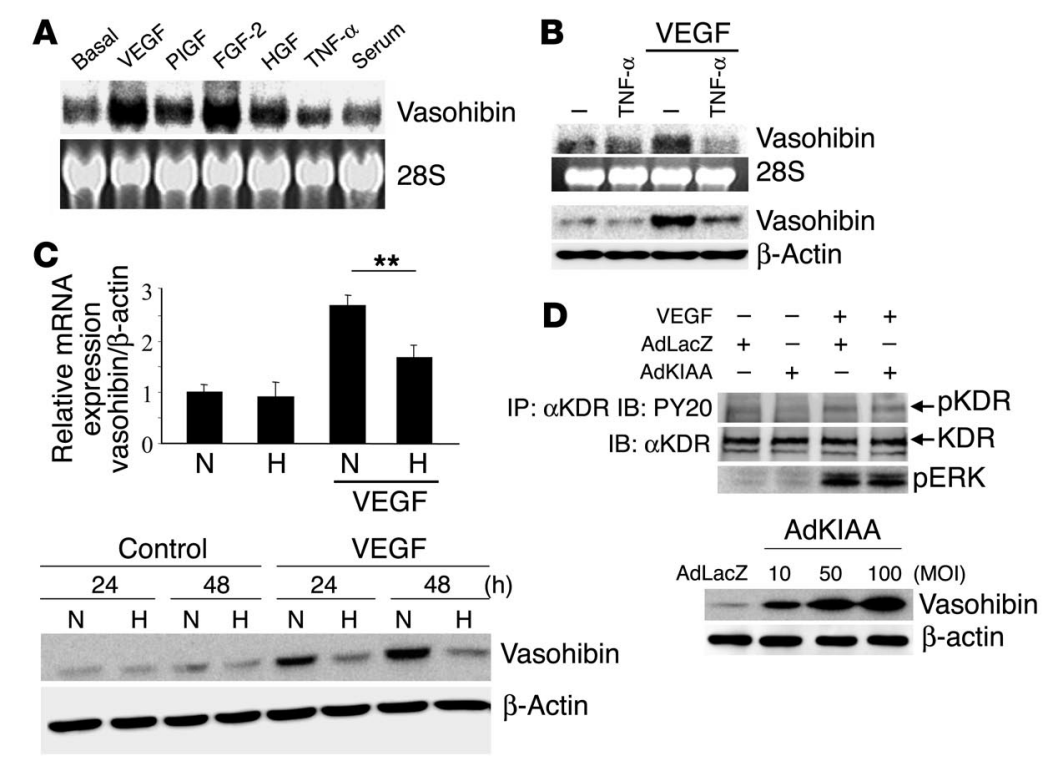

Figure 5

Modulation of vasohibin expression and the effect of vasohibin on VEGF-stimulated signaling in HUVECs. (A) Induction of vasohibin. HUVECs were stimulated with VEGF (1 nM), PIGF (1 nM), FGF-2 (2 nM), HGF (1 nM), TNF- $\alpha(1 \mathrm{nM})$, or $10 \%$ serum for 24 hours. Thereafter, total RNA was obtained and Northern blotting for vasohibin was performed. (B) Effect of TNF- $\alpha$ on the induction of vasohibin by VEGF. HUVECs were stimulated with VEGF $(1 \mathrm{nM})$ and/or TNF- $\alpha(1 \mathrm{nM})$. Thereafter, Northern blotting and Western blotting for vasohibin were performed. (C) Effect of hypoxia on the induction of vasohibin by VEGF. HUVECs were stimulated with VEGF (1 nM) under normoxic $(\mathrm{N})$ or hypoxic $(\mathrm{H})$ conditions. Upper panel: Total RNA was obtained and real-time RT-PCR of vasohibin was performed. Values are expressed as mean \pm SD of 4 samples. ${ }^{\star \star} P<0.01$. Lower panel: Cell extract was obtained and Western blotting for vasohibin was performed. (D) Effect of vasohibin on VEGF-mediated KDR tyrosine phosphorylation or ERK1/2 activation of HUVECs. HUVECs were infected with AdLacZ or AdKIAA at an MOI of 100 , and then stimulated with VEGF $(10 \mathrm{ng} / \mathrm{ml})$. VEGF-mediated KDR tyrosine phosphorylation or ERK1/2 activation was analyzed. Results shown in lower panel indicate that AdKIAA increased the synthesis of vasohibin in an MOI-dependent manner. IP, immunoprecipitation; IB, immunoblotting; pKDR, phosphorylated KDR. 
A

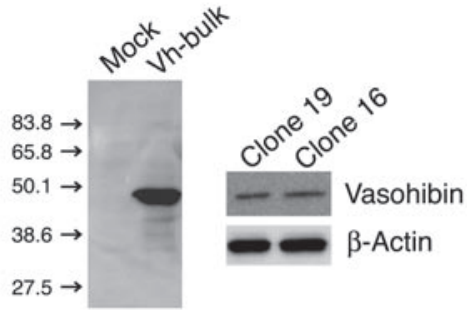

C

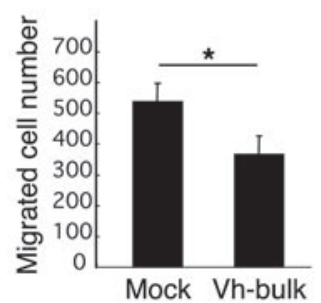

E

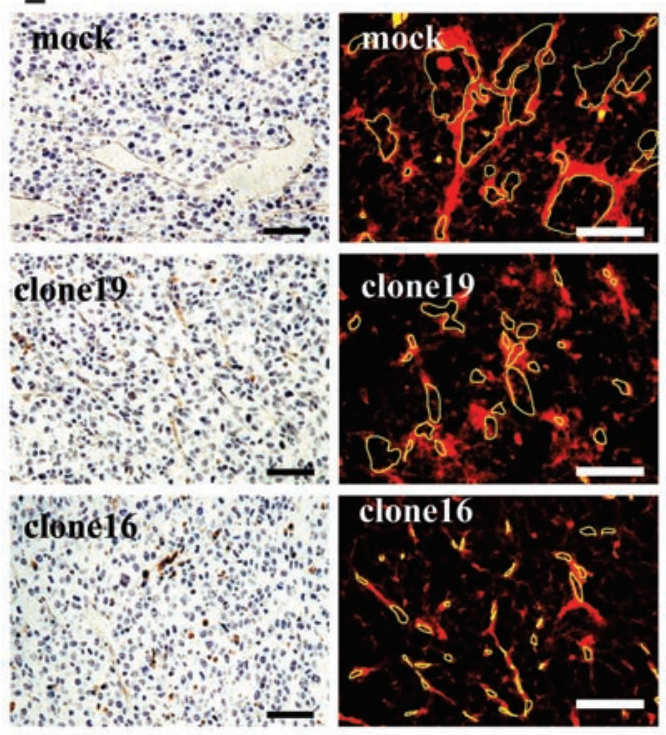

B
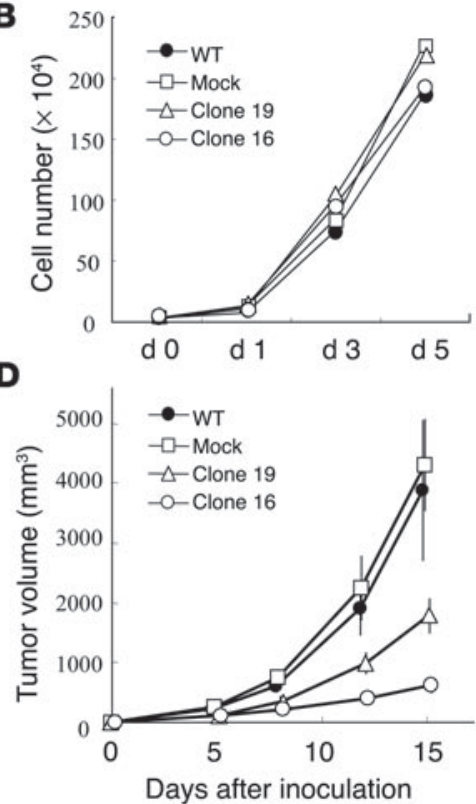

$\mathbf{F}$

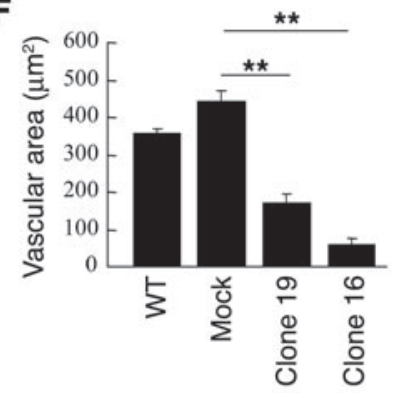

Figure 6

Vasohibin suppresses tumor growth and tumor angiogenesis. (A) The synthesis of vasohibin protein in LLC cells. Cell extracts were prepared from mock or vasohibin transfectants (Vh-bulk) for Western blotting. Clone 16 and clone 19 were vasohibinproducing clones. (B) Effect of vasohibin on the proliferation of LLC cells in vitro. Proliferation of mock transfectants, vasohibin transfectants, clone 16, and clone 19 was determined. (C) Effect of secreted vasohibin from LLC cells on the migration of HUVECs. Mock or vasohibin transfectants were plated on the lower compartment of a modified Boyden chamber and the migration of HUVECs toward the lower chamber of the Transwell insert was analyzed. Values are expressed as mean \pm SD of 4 samples. (D) Effect of vasohibin gene transfection on the growth of LLC cells in vivo. BDF1 mice were inoculated intradermally with LLC cells. Tumor volume was determined consecutively. (E) Effect of vasohibin gene transfection on tumor angiogenesis. Paraffin sections were prepared from tumors for the immunostaining of CD31; sections obtained on day 8 after inoculation are shown. Visualization with a DAKO LSAB+/HRP kit is shown at left, and that with streptavidinСy3 conjugate on the right. Yellow lines trace vascular lumens. Scale bars: $50 \mu \mathrm{m}$. (F) Quantitative analysis of tumor vascular area. Total vascular area per field was determined using NIH Image and compared. Values are expressed as mean \pm SD of 6 random fields. ${ }^{\star} P<0.05 ;{ }^{* *} P<0.01$. inhibited tumor growth and tumor angiogenesis very efficiently. These results suggest that exogenous vasohibin gene transfection or vasohibin protein administration might be powerful tools for the treatment of tumor angiogenesis.

In conclusion, we have isolated and characterized a novel endothelium-derived angiogenesis inhibitor, vasohibin. Our data indicate that vasohibin is the first candidate negative feedback regulator of angiogenesis. We believe that our present study will guide us to a novel research field of angiogenesis and vascular development.

\section{Methods}

Materials. The materials used and their sources were as follows: VEGF 165 and HGF (Sigma-Aldrich); FGF-2 and growth factor-reduced Matrigel (Collaborative Research Inc.); PlGF and TNF- $\alpha$ (R\&D Systems Inc.); a-MEM, DMEM, and medium 199 (M199; Nissui Pharmaceutical Co.); endothelial basal medium (EBM) containing EC growth supplements (Clonetics Corp.); FBS (JRH Biosciences Inc.); Opti-MEM, Lipofectin, and Oligofectamine (Invitrogen Corp.); ISOGEN (Nippon Gene Co.); Hybond $\mathrm{N}^{+}$ membrane, nitrocellulose membranes, and protein A Sepharose (Amersham
Biosciences); mouse anti-human CD31 mAb (DakoCytomation); rat antimouse CD31 Ab (Research Diagnostics Inc.); and biotin-conjugated antirabbit IgG (Vector Laboratories Inc.). Unless indicated, all other chemicals were purchased from Sigma-Aldrich.

Cells and cell culture. HUVECs, HAECs, HMVECs, HASMCs, human fibroblasts, and human keratinocytes were obtained from Kurabo Industries Ltd. HUVECs, HAECs, and HMVECs were routinely cultured on type I collagen-coated Iwaki dishes (Asahi Techno Glass) in EBM containing EC growth supplements and 10\% FBS. HASMCs were cultured in HuMedia SG2 with growth supplement for SMCs (Kurabo Industries Ltd.), human fibroblasts in 106S medium with low-serum growth supplement (Kurabo Industries Ltd.), and human keratinocytes in HuMedia KG2 with growth supplement for keratinocytes (Kurabo Industries Ltd.). Chemically transformed bovine ECs, GM7373 cells, were obtained from American Type Culture Collection and were grown in DMEM and 10\% FBS.

For the hypoxia experiment, HUVECs were placed in a hypoxia chamber (Bellco Glass Inc.) containing $0 \% \mathrm{O}_{2}, 5 \% \mathrm{CO}_{2}$, and $95 \% \mathrm{~N}_{2}$. The chamber was maintained at $37^{\circ} \mathrm{C}(27)$.

Preparation of anti-KIAA1036 $\mathrm{mAb}$. A/J mice (Japan SLC Inc.) were immunized 3 times with Cys-KIAA1036 peptide (Gly286-Arg299 of KIAA1036 
protein) and keyhole limpet hemocyanin conjugate. Spleen cells from the immunized mice were fused with myeloma cells (P3U1) by the method described previously (28). The culture supernatant of each hybridoma was screened as follows. Microplates (module plate F8, Nalge Nunc International) were coated with goat anti-mouse IgG. Hybridoma supernatants and a mixture of the biotinylated KIAA1036 protein and europium-labeled streptavidin were added to the wells and incubated at room temperature for 2 hours or at $4{ }^{\circ} \mathrm{C}$ overnight. After the wells were washed 3 times with saline containing $0.01 \%$ Tween 20 and $0.02 \% \mathrm{NaN}_{3}, 100 \mu \mathrm{l}$ DELFIA Enhancement Solution (PerkinElmer Life Sciences - Wallac Oy) was added to each well and mixed for 5 minutes. Then the time-resolved fluorescence at $615 \mathrm{~nm}$ was measured in each well. Positive hybridomas were cloned by a limiting dilution technique. The resulting $\mathrm{mAb}$ (IgG2a, subclass $\kappa$ ) was purified from ascites by protein A-agarose affinity chromatography (MAPS II kit, Bio-Rad Laboratories Inc.).

Northern blot analysis. Northern blotting was performed as described (29). Briefly, cells were starved in $0.1 \% \mathrm{FBS} / \alpha$-MEM for 12 hours, and then stimulated with a growth factor or a cytokine for the indicated period. Total RNA was extracted by ISOGEN according to the manufacturer's instructions and was separated on a $1 \%$ agarose gel containing $2.2 \mathrm{M}$ formaldehyde and transferred to a Hybond $\mathrm{N}^{+}$membrane. For analysis of tissue distribution, Multiple Tissue Northern Blot (BD Biosciences - Clontech) or a human embryonic message HUNTER (Geno Technology Inc.) was used. The membrane was hybridized with a ${ }^{32}$ P-labeled KIAA1036 (vasohibin) cDNA probe containing an open reading frame (464-1,417 bp). Autoradiography was carried out on an imaging plate and analyzed with a fluorescent image analyzer (FLA-2000; Fuji Photo Film Co.) and a luminescent image analyzer (LAS-1000; Fuji Photo Film Co.).

Western blot analysis. Cells were lysed with modified RIPA buffer $(2 \mathrm{mM}$ sodium orthovanadate, $50 \mathrm{mM} \mathrm{NaF}, 20 \mathrm{mM}$ HEPES, $150 \mathrm{mM} \mathrm{NaCl}, 1.5$ $\mathrm{mM} \mathrm{MgCl} 2,5 \mathrm{mM}$ sodium pyrophosphate, $10 \%$ glycerol, $0.2 \%$ Triton $\mathrm{X}-100,5 \mathrm{mM}$ EDTA, $1 \mathrm{mM}$ PMSF, $10 \mu \mathrm{g} / \mathrm{ml}$ leupeptin, and $10 \mu \mathrm{g} / \mathrm{ml}$ aprotinin) as previously described (30). Cell extract was then separated by SDS-PAGE on a $10 \%$ separating gel and transferred to nitrocellulose membranes. The membranes were blocked for 1 hour at room temperature with Tris-buffered saline (TBS, pH 7.6) containing 5\% skim milk, and then incubated for 1 hour at room temperature in TBS containing $0.05 \%$ Tween 20 (T-TBS), 2.5\% skim milk, and $1 \mu \mathrm{g} / \mathrm{ml} \mathrm{HRP-conjugated} \mathrm{anti-}$ KIAA1036 mAb. After the filters were washed 3 times with T-TBS, blots were detected using an enhanced chemiluminescence method, the ECL Western blotting detection kit from Amersham Biosciences. The results were visualized using an LAS-1000 (Fuji Photo Film Co.).

Immunoprecipitation and Western blotting for secreted KIAA1036/vasobibin. Subconfluent HUVECs were cultured for 3 days in EBM containing EC growth supplements and $10 \%$ FBS. The medium was collected and concentrated with an Amicon Ultra-4 (10,000 MWCO; Millipore Corp.). Centrifugation at 2,300 $\mathrm{g}$ for 20 minutes resulted in 15 -fold increased concentration. Five hundred microliters of concentrated medium was incubated with $2 \mu \mathrm{g}$ of anti-KIAA1036 mAb overnight at $4^{\circ} \mathrm{C}$. Twenty microliters of protein A Sepharose was added, followed by agitation for 2 hours. Western blotting of the immunoprecipitated protein was carried out as described above.

Quantitative RT-PCR. HUVECs were preincubated in $0.1 \%$ FBS $/ \alpha-M E M$ for 12 hours, and then stimulated with the indicated concentrations of VEGF for 24 hours. Total RNA was extracted by ISOGEN according to the manufacturer's instructions. First-strand cDNA was generated using a first-strand cDNA synthesis kit for RT-PCR (Roche Diagnostics Corp.). Quantitative RT-PCR was performed with a LightCycler system (Roche Diagnostics Corp.) according to the manufacturer's instructions. The amount of PCR product was measured as a fluorescence signal proportional to the amount of the specific target sequence present. The sense and antisense primer pairs used were: KIAA1036 (vasohibin), 5'AGATCCCCATACCGAGTGTG-3' and 5'-GGGCCTCTTTGGTCATTTCC$3^{\prime}$; and $\beta$-actin, $5^{\prime}$-ACAATGAGGTGCGTGTGGCT-3' and $5^{\prime}$-TCTCCTTAATGTCACGCACGA-3' ${ }^{\prime}$, respectively.

Immunofluorescence staining of ER and KIAA1036 in HUVECs. HUVECs were fixed with methanol at $-20^{\circ} \mathrm{C}$ and permeabilized with $0.1 \%$ NP-40 in PBS. Nonspecific binding sites were blocked with $1 \% \mathrm{BSA}$ and $1.5 \%$ goat serum in PBS. ER was detected by indirect immunofluorescence using anti-calnexin $\mathrm{Ab}$ (Santa Cruz Biotechnology Inc.) and rhodamine-conjugated anti-rabbit IgG Ab (Chemicon International Inc.). KIAA1036 was detected using anti-KIAA1036 $\mathrm{mAb}$ and FITC-conjugated goat anti-mouse IgG Ab (Jackson ImmunoResearch Laboratories). The cells were observed by confocal microscopy (LSM 5 PASCAL, Carl Zeiss Jena GmbH).

Preparation of KIAA1036 protein. Original full-length KIAA1036 cDNA (clone FH01447) was obtained from the Kazusa DNA Research Institute. Using this cDNA as a template, a cDNA fragment corresponding to the entire open reading frame was amplified with the oligonucleotide primers 5'-CCGCGGCCGCCGAAGATTTAGGGATGCCAG-3' and 5'-CATCTAGATCCGACCCGGATCTGGTACC-3' by PCR, adding a NotI site at the $5^{\prime}$ end, an XbaI site at the $3^{\prime}$ end, and the termination codon substituted with a glycine codon. This fragment was cloned into the NotI and XbaI sites in expression vector $\mathrm{p} 3 \mathrm{xFLAG-CMV-14}$ (Sigma-Aldrich) to generate pFLAG14-KIAA1036, in which KIAA1036 cDNA was connected to a triple repeat of the FLAG tag sequence at the $3^{\prime}$ end. The DNA fragment containing the KIAA 1036 cDNA joined with $3 x$ FLAG was then cut off at the NotI and Eam1105I sites (followed by substitution of the XhoI site) of pFLAG14-KIAA1036 and cloned into the NotI and XhoI sites of vector pFastBac1 (Invitrogen Corp.). Using this plasmid, pFastBac1-KIAA1036, the KIAA1036 protein connected to the FLAG tag at the C-terminus was expressed in a Bac-to-Bac baculovirus expression system (Invitrogen Corp.) according to the manufacturer's instructions. Transfected Sf9 cells were cultivated as a source of recombinant baculovirus and the conditioned medium was substantially infected with freshly cultured Sf9 cells. After a 96-hour incubation at $28^{\circ} \mathrm{C}$, transfected Sf9 cells were harvested and suspended in $50 \mathrm{mM}$ Tris- $\mathrm{HCl}$ ( $\mathrm{pH}$ 7.4), $0.15 \mathrm{M} \mathrm{NaCl}, 0.1 \mathrm{mM}$ EDTA, 0.1 mM EGTA, 1 mM DTT, 0.1 mM amidinophenyl methanesulfonyl fluoride hydrochloride, and $0.1 \% \mathrm{NP}-40$, then sonicated. After centrifugation, the soluble fraction was collected and applied to an anti-FLAG M2 affinity column (Sigma-Aldrich). After washing with TBS (pH 7.4) and 0.1\% NP-40, the KIAA1036 protein was eluted from the column with $100 \mathrm{mM}$ glycine$\mathrm{HCl}(\mathrm{pH} 3.5$ ) and $0.1 \% \mathrm{NP}-40$ and neutralized with $1 \mathrm{M}$ Tris- $\mathrm{HCl}$ ( $\mathrm{pH} 8.0$ ).

Network formation by HUVECs. Ice-cold growth factor-reduced Matrigel was poured into a $35-\mathrm{mm}$ dish and allowed to gel for 1 hour at $37^{\circ} \mathrm{C}$. Thereafter, HUVECs $\left(5 \times 10^{4}\right)$ were plated onto Matrigel and incubated with the KIAA1036 protein $(10 \mathrm{nM})$ or vehicle $(20 \mathrm{mM}$ Tris $/ \mathrm{HCl}, 0.1 \%$ NP-40, and 0.1 M glycine, pH 7.0) in M199 containing 5\% FBS. After a 9hour incubation, cells were photographed.

Matrigel implantation assay. All the animal studies were reviewed and approved by the committee for animal study at our institute in accord with established standards of humane handling. The Matrigel implantation assay was performed as described previously (31). Briefly, $200 \mu \mathrm{l}$ of growth factor-reduced Matrigel containing VEGF (100 ng/ml) plus heparin (32 U/ $\mathrm{ml})$ and/or KIAA1036/vasohibin $(10 \mathrm{nM})$ in liquid form at $4{ }^{\circ} \mathrm{C}$ was injected into the abdominal subcutaneous tissue of the midperitoneal region of each mouse. On day 7 after injection, the mice were sacrificed and gels were recovered. The gels were fixed in $4 \%$ paraformaldehyde in PBS and embedded in paraffin. Three micrometer sections of the gels were subjected to histological analysis. Immunohistochemical analysis revealed that almost all of the cells that had entered the Matrigel were ECs (31). The vessel number per low-power field in 3 different fields was counted for each sample. 
Mouse corneal micropocket assay. All the animal studies were reviewed and approved by the committee for animal study in our institute in accord with established standards of humane handling. The mouse corneal micropocket assay and quantification of corneal neovascularization was performed according to the method described by Ogawa et al. (32). Briefly, $0.3 \mu \mathrm{l}$ of Hydron pellets (IFN Sciences) containing FGF-2 (80 ng) with or without KIAA1036 protein ( $4 \mathrm{ng}$ ) was implanted in the corneas of BALB/c mice (Charles River Japan). Seven days after the implantation, animals were sacrificed and the corneal vessels were photographed. Quantification of neovascularization in cornea was performed using NIH Image software.

Adenovirus vector. A replication-defective adenovirus vector encoding KIAA1036 (AdKIAA) was prepared according to the method described previously (33). A replication-defective adenovirus vector encoding the $\beta$-gal gene (AdLacZ) was used to control for the nonspecific effects of viral infection (33). Plaque-purified adenoviruses were propagated in HEK 293 cells. The viral lysates were purified and concentrated through 2 cycles of $\mathrm{CsCl}$ step gradients.

CAM assay. A CAM assay using adenovirus vectors was performed as described by Schughart and Accart (34) with some modification. On day 0 , the incubation of fertilized chick eggs was started in a humidified chamber at $37^{\circ} \mathrm{C}$. On day 2 , a window was cut into each eggshell and the underlying membrane, and $1.5 \mathrm{ml}$ of the white was aspirated from each egg with an 18gauge needle. The eggs were incubated again for 4 hours with the windows sealed. Adenovirus vector (AdLacZ or AdKIAA) was mixed with growth factor-reduced Matrigel on ice and $10 \mu \mathrm{l}$ of the mixture was placed on a sterilized $3 \mathrm{~mm} \times 3 \mathrm{~mm}$ nylon mesh (pore size $300 \mu \mathrm{m}$ ) (Sefar Nitex 03-300/51; Sefar Inc.). The adenovirus/Matrigel mixture on the mesh was then placed on a piece of Parafilm and incubated in a humidified chamber at $37^{\circ} \mathrm{C}$ for 30 minutes to allow gel formation. The nylon mesh support containing the adenovirus/Matrigel mixture $\left(1 \times 10^{8} \mathrm{PFU} / \mathrm{CAM}\right)$ was placed onto the CAM, where no vascular structure was yet visible. A quarter piece of a sterilized plastic (Thermanox) cover slip (13 mm diameter; Nalge Nunc International) was put on top of the mixture to avoid drying the gel and the eggs were incubated for another 2 days. On day 4 , vascular formation was evaluated by macroscopic observation and photographs were taken at $\times 8.0$ magnification (Leica MZ FLIII, Leica Microsystems).

Cell migration. The indicated concentrations of growth factors and/or the KIAA1036 protein in M199 containing 5\% FBS were placed in the lower chamber of Transwell inserts with 6.5 - $\mu \mathrm{m}$ pores. HUVECs $\left(5 \times 10^{4}\right)$ in M199 containing $5 \%$ FBS were plated in the upper chamber (Corning-Costar Corp.) and allowed to migrate for 4 hours at $37^{\circ} \mathrm{C}$. The number of cells that migrated across the filter was counted in 4 high-power $(\times 200)$ fields per insert. The concentrations of growth factors were chosen to obtain maximum effect.

BrdU uptake. HUVECs $\left(3 \times 10^{3}\right)$ were plated in a 96-well type I collagen-coated plate and incubated in EBM containing 10\% FBS for 24 hours. Thereafter, HUVECs were incubated in M199 containing 5\% FBS for 24 hours, and then stimulated with the indicated concentrations of growth factors and/or KIAA1036 protein for 12 hours. BrdU uptake was analyzed by Cell Proliferation ELISA (Roche Diagnostics Corp.) according to the manufacturer's protocol. The concentrations of growth factors were chosen to obtain maximum effect.

ODN treatment. HUVECs were rinsed with Opti-MEM and incubated for 4 hours with $500 \mathrm{nM}$ synthetic phosphorothioate ODNs in Oligofectamine containing Opti-MEM. Thereafter, HUVECs were harvested and resuspended in M199 containing 5\% FBS with or without VEGF $(50 \mathrm{ng} / \mathrm{ml})$, and the Transwell migration assay was carried out as described above. The sequences of ODNs were: AS-ODN, 5'-CCCCCTGGCATCCCTAAATC$3^{\prime}$ (which is complementary to the mRNA region in KIAA1036); S-ODN, 5'-GATTTAGGGATGCCAGGGGG-3'; and Scr-ODN, 5'-ATCCTGCCCGCAACCTCATC- $3^{\prime}$, comprising all the bp's of the antisense codons in ran- dom order. AS-ODN was not homologous to any genes checked against the reported DNA sequences present in the BLAST program.

Immunohistochemical analysis of human placenta. Mouse anti-human CD31 $\mathrm{mAb}$, anti-KIAA1036 $\mathrm{mAb}$, or mouse IgG was used as the primary Ab. These were diluted 300- to 600-fold with PBS containing $1 \%$ BSA. The secondary $\mathrm{Ab}$ was biotin-conjugated anti-rabbit $\mathrm{Ab}$ diluted 300 -fold with the same diluent. Each reaction was conducted for 60 minutes at room temperature. Coupling of streptavidin to biotin using an $\mathrm{ABC}$ kit (Vector Laboratories Inc.) was performed for 45 minutes at room temperature. Immunocomplexes were visualized with 3,3'-diamidobenzidine tetrahydrochloride tablet sets (Sigma-Aldrich).

Tyrosine phosphorylation of KDR and activation of ERK1/2. HUVECs were infected with AdLacZ or AdKIAA at an MOI of 100, and then subjected to the following procedure. For tyrosine phosphorylation of KDR, HUVECs were incubated with VEGF $(10 \mathrm{ng} / \mathrm{ml})$ for 5 minutes. Samples were then prepared as described in Western blot analysis and incubated with antifetal liver kinase-1 (anti-Flk-1)/KDR Ab (Santa Cruz Biotechnology Inc.) and protein A Sepharose overnight at $4{ }^{\circ} \mathrm{C}$. The immunoprecipitates were washed 4 times in modified RIPA buffer, boiled for 5 minutes in reducing buffer, and subjected to SDS-PAGE. Western blotting was then performed using PY20 Ab (Santa Cruz Biotechnology Inc.) or anti-Flk-1/KDR Ab as primary $\mathrm{Ab}$. For the activation of ERK1/2, HUVECs were incubated with VEGF $(10 \mathrm{ng} / \mathrm{ml})$ for 15 minutes. Samples were then prepared, and Western blotting was performed using anti-phospho-ERK1/2 (Cell Signaling Technology) as a primary Ab.

Establishment of permanent vasobibin gene transfectants. LLC cells were obtained from the National Cancer Institute. LLC cells or GM7373 cells were transfected with p3xFLAG-CMV-14 alone or pFLAG14-KIAA1036 with FuGENE6 reagent. After a 48-hour incubation, $1 \mathrm{mg} / \mathrm{ml} \mathrm{G} 418$ was added to the medium to select for stable transfectants. After several passages, more than 100 G418-resistant colonies were combined and defined as bulk transfectants. Thereafter, transfected LLC cells were subjected to clonal selection in the presence of G418. After several weeks, colonies formed on the culture dish. Twenty-four of these colonies were picked up and expanded. Of these colonies, we selected 2 clones (clone 16 and clone 19) that stably expressed a fair amount of KIAA1036 protein.

Tumor growth and tumor angiogenesis in vivo. All the animal studies were reviewed and approved by the committee for animal study at our institute in accord with established standards of humane handling. Experiments consisted of 10 mice per group. Female BDF1 mice, 6-7 weeks old (Japan SLC Inc.), were inoculated intradermally with $5 \times 10^{5}$ transfected LLC cells. Tumor size was evaluated periodically. At day 8 and day 12, 2 animals were sacrificed, and paraffin sections were prepared from the excised tumors. Tissue sections were examined with rat anti-mouse $\mathrm{CD} 31 \mathrm{Ab}$ at a dilution of 1:25, and labeling was visualized with a DAKO LSAB+/HRP kit (DakoCytomation) with 3,3'-diaminobenzidine tetrahydrochloride. Sections were counterstained with hematoxylin. Alternatively, tissue sections were pretreated with $0.4 \mathrm{mg} / \mathrm{ml}$ proteinase $\mathrm{K}$ in 0.05 Tris- $\mathrm{HCl}$ buffer ( $\mathrm{pH} 7.6$ ) for 15 minutes. Endogenous peroxidase was quenched with $3 \% \mathrm{H}_{2} \mathrm{O}_{2}$ for 5 minutes. Sections were blocked for 10 minutes with Non-Specific Staining Blocking Reagent (DakoCytomation). The blocking reagent was removed and the tissue was exposed to the primary $\mathrm{Ab}$ overnight at $4{ }^{\circ} \mathrm{C}$. The next day, the tissue was washed with TBS ( $\mathrm{pH}$ 7.6) and exposed to the secondary $\mathrm{Ab}$, rabbit anti-rat IgG/biotin (DakoCytomation) at a dilution of 1:400 for 30 minutes. Labeling was visualized using a streptavidin-Cy3 conjugate (Sigma-Aldrich) at a dilution of 1:100. The vascular lumen was traced and vascular luminal area was analyzed with NIH Image.

Calculations and statistical analysis. The statistical significance of differences in the data was evaluated by unpaired ANOVA, and $P$ values were calculated using the unpaired Student's $t$ test. 


\section{Acknowledgments}

We thank Takuya Moriya for comments and Kevin Boru for review of the manuscript. We also thank Kyoko Shimizu for her excellent technical assistance. This work was supported by the Japan Society of the Promotion of Science Research for the Future (grant 99L01304).

Received for publication January 26, 2004, and accepted in revised form July 27, 2004.

1. Folkman, J. 1995. Angiogenesis in cancer, vascular, rheumatoid and other disease. Nat. Med. 1:27-31.

2. Ribatti, D., Vacca, A., and Presta, M. 2000. The discovery of angiogenic factors: a historical review. Gen. Pharmacol. 35:227-231.

3. Ferrara, N. 2002. VEGF and the quest for tumour angiogenesis factors. Nat. Rev. Cancer. 2:795-803.

4. Good, D.J., et al. 1990. A tumor suppressor-dependent inhibitor of angiogenesis is immunologically and functionally indistinguishable from a fragment of thrombospondin. Proc. Natl. Acad. Sci. U. S. A 87:6624-6628

5. Dameron, K.M., Volpert, O.V., Tainsky, M.A., and Bouck, N.P. 1994. Control of angiogenesis in fibroblasts by p 53 regulation of thrombospondin-1. Science. 265:1582-1584.

6. Tombran-Tink, J., Chader, G.G., and Johnson, L.V. 1991. PEDF: a pigment epithelium-derived factor with potent neuronal differentiative activity. Exp. Eye Res. 53:411-414.

7. Dawson, D.W., et al. 1999. Pigment epitheliumderived factor: a potent inhibitor of angiogenesis. Science. 285:245-248.

8. Ferrara, N., Clapp, C., and Weiner, R. 1991. The 16K fragment of prolactin specifically inhibits basal or fibroblast growth factor stimulated growth of capillary endothelial cells. Endocrinology. 129:896-900.

9. O’Reilly, M.S., et al. 1994. Angiostatin: a novel angiogenesis inhibitor that mediates the suppression of metastases by a Lewis lung carcinoma. Cell. 79:315-328.

10. O'Reilly, M.S., et al. 1997. Endostatin: an endogenous inhibitor of angiogenesis and tumor growth. Cell. 88:277-285.

11. Cao, Y., Chen, C., Weatherbee, J.A., Tsang, M., and Folkman, J. 1995. gro- $\beta$, a -C-X-C-chemokine, is an angiogenesis inhibitor that suppresses the growth of Lewis lung carcinoma in mice. J. Exp. Med. 182:2069-2077.

12. Strieter, R.M., Kunkel, S.L., Arenberg, D.A., Burdick,
Address correspondence to: Yasufumi Sato, Department of Vascular Biology, Institute of Development, Aging, and Cancer, Tohoku University, 4-1, Seiryo-machi, Aoba-ku, Sendai 980-8575, Japan. Phone: 81-22-717-8528; Fax: 81-22-717-8533; E-mail: y-sato@idac.tohoku.ac.jp.

Keiichi Imagawa's present address is: First Department of Internal Medicine, Nara Medical University, 840 Shijo-cho, Kashihara 634-8521, Japan.
M.D., and Polverini, P.J. 1995. Interferon $\gamma$-inducible protein 10 (IP-10), a member of the C-X-C chemokine family, is an inhibitor of angiogenesis. Biochem. Biophys. Res. Commun. 210:51-57.

13. Maione, T.E., et al. 1990. Inhibition of angiogenesis by recombinant human platelet factor-4 and related peptides. Science. 247:77-79.

14. Yancopoulos, G.D., et al. 2000. Vascular-specific growth factors and blood vessel formation. Nature. 407:242-248.

15. Lord, B.I. 1988. Feedback regulators in normal and tumour tissues. J. Cell Sci. Suppl. 10:231-242.

16. Sargiannidou, L., Zhou, J., and Tuszynski, G.P. 2001. The role of thrombospondin-1 in tumor progression. Exp. Biol. Med. 226:726-733.

17. Doll, J.A., et al. 2003. Pigment epithelium-derived factor regulates the vasculature and mass of the prostate and pancreas. Nat. Med. 9:774-780.

18. Folkman, J. 2002. Role of angiogenesis in tumor growth and metastasis. Semin. Oncol. 6:15-18.

19. Abe, M., and Sato, Y. 2001. cDNA microarray analysis of the gene expression profile of VEGFactivated human umbilical vein endothelial cells. Angiogenesis. 4:289-298.

20. Kanno, S., et al. 2000. Roles of two VEGF receptors, Flt-1 and KDR, in the signal transduction of VEGF effects in human vascular endothelial cells. Oncogene. 19:2138-2146.

21. Pugh, C.W., and Ratcliffe, P.J. 2003. Regulation of angiogenesis by hypoxia: role of the HIF system. Nat. Med. 9:677-684.

22. Harris, A.L. 2002. Hypoxia--a key regulatory factor in tumour growth. Nat. Rev. Cancer. 2:38-47.

23. Key, M.E. 1983. Macrophages in cancer metastases and their relevance to metastatic growth. Cancer Metastasis Rev. 2:75-88.

24. Kobayashi, Y., et al. 1990. Identification of calciumactivated neutral protease as a processing enzyme of human interleukin $1 \alpha$. Proc. Natl. Acad. Sci. U. S. A. 87:5548-5552.
25. Watanabe, N., and Kobayashi, Y. 1994. Selective release of a processed form of interleukin $1 \alpha$. Cytokine. 6:597-601.

26. Wilson, K.P., et al. 1994. Structure and mechanism of interleukin-1 $\beta$ converting enzyme. Nature. 370:270-275.

27. Oikawa, M., et al. 2001. Hypoxia induces transcription factor ETS-1 via the activity of hypoxiainducible factor-1. Biochem. Biophys. Res. Commun. 289:39-43.

28. Ohta, H., et al. 1999. One-step direct assay for mature-type adrenomedullin with monoclonal antibodies. Clin. Chem. 45:244-251.

29. Iwasaka, C., Tanaka, K., Abe, M., and Sato, Y. 1996. Ets-1 regulates angiogenesis by inducing the expression of urokinase-type plasminogen activator and matrix metalloproteinase- 1 and the migration of vascular endothelial cells. J. Cell. Physiol. 169:522-531.

30. Tanaka, K., Oda, N., Iwasaka, C., Abe, M., and Sato, Y. 1998. Induction of Ets- 1 in endothelial cells during re-endothelialization after denuding injury. J. Cell. Physiol. 176:235-244.

31. Miyashita, H., et al. 2002. A mouse orthologue of puromycin-insensitive leucyl-specific aminopeptidase is expressed in endothelial cells and plays an important role in angiogenesis. Blood. 99:3241-3249.

32. Ogawa, S., et al. 1999. Induction of macrophage inflammatory protein-1alpha and vascular endothelial growth factor during inflammatory neovascularization in the mouse cornea. Angiogenesis. 3:327-334.

33. Nakano, T., et al. 2000. Angiogenesis inhibition by transdominant mutant Ets-1. J. Cell. Physiol. 184:255-262

34. Schughart, K., and Accart, N. 2003. Use of adenovirus vectors for functional gene analysis in the chicken chorioallantoic membrane. Biotechniques. 34:178-183. 